\title{
Xerostomia as a Contributing Factor for Taste Distortion in Patients Undergoing Chronic Dialysis Treatment
}

\author{
D. Konstantinova ${ }^{1}$, A. Nenova-Nogalcheva ${ }^{2}$, P. Pechalova ${ }^{3}$, R. Pancheva ${ }^{4}$, D. Andonova ${ }^{5}$ \\ ${ }^{1}$ Department of Prosthetic Dentistry, Faculty of dental medicine, Medical University - Varna, Bulgaria \\ ${ }^{2}$ Department of Oral Surgery, Faculty of dental medicine, Medical University - Varna, Bulgaria \\ ${ }^{3}$ Department of Oral Surgery, Faculty of dental medicine, Medical University - Plovdiv, Bulgaria \\ ${ }^{4}$ Department of Hygiene, Disaster Medicine and Epidemiology, Medical University - Varna, Bulgaria \\ ${ }^{5}$ Department of Psychology, Varna Free University, Bulgaria \\ pechalova@abv.bg
}

\begin{abstract}
Xerostomia is defined as the subjective sensation of dry mouth. Although xerostomia is a subjective sensation, decreased amount of saliva could worst oral health and could be a reason of difficulty in chewing, swallowing and speaking, as well as could be a reason of development and enlargement of lesions on the oral mucosa, particularly on the periodontium and the tongue, increased incidence of dental caries and presents of odor.

The aim of the present study was to determine the prevalence of the subjective sensation of dry mouth in patients with end-stage chronic kidney disease and in healthy control patients, using a questionnaire.

Material and methods: The clinical study involved 104 patients divided into 2 groups: a Control Group, comprising 34 people without any common diseases and a Clinical Group, consisting of 70 patients with endstage chronic kidney disease (CKD) undergoing renal replacement therapy. The data was gathered using a questionnaire listing questions related to the subjective sense of dry mouth, taste distortion, burning mouth, loss of taste, soreness while eating and swallowing.

Results: The analysis of the results indicated a statistically significant relationship between dry mouth complaints and the patients' group affiliation $\left(\chi^{2}=34.47, p=0.000\right)$. A statistically significant dependence between patients' age and dry mouth symptoms was not detected, however a tendency towards a greater number of dry mouth complaints among patients over 60 years of age was apparent $\left(\chi^{2}=2.08, p=0.149\right)$. The observations revealed significant positive correlations between dry mouth and respectively, taste distortion, metallic taste, loss of taste, soreness while eating/swallowing as well as unpleasant breath. There was a statistical dependence between xerostomia and loss of taste, and between xerostomia and soreness while swallowing among patients who had been on renal replacement treatment for over 5 years. Statistical correlation was also observed between the subjective sense of mouth dryness and unpleasant breath in patients who had been treated for less than 5 years.

Conclusion: Chronic kidney disease has a lasting and significant impact on patients' quality of life and poses a serious health and social problem. Although xerostomia is a subjective sensation, its manifestation in patients on hemodialysis can affect their oral health, which in turn often leads to difficulty in chewing, swallowing and speaking. The issue is still relevant with the increasing incidence of CKD and its relation the quality of life of this group of patients.
\end{abstract}

Keywords: hemodialysis, taste distortion, xerostomia

www.arjonline.org

Page 1 


\section{INTRODUCTION}

The primary role of saliva is to build a protective film over the oral mucosa and the dental tissues owing to the mucus contained in it. By the action of key digestive enzymes such as amylase and lipase, saliva is actively involved in the formation of the nutritional bolus, thus assisting swallowing and digestion. Saliva breaks down sugars and fats and helps trigger taste receptors. A decrease in salivary flow rate may lead to a transient or chronic sensation of dry mouth. The incidence of xerostomia is commonly linked to various factors such as fear, tobacco, all causes of extracellular dehydration, immediate effects of toxic drugs, sepsis (viral or bacterial), allergic conditions, etc. The causes of dry mouth can be identified at the three levels of saliva production: deficiency of water or necessary metabolites (dehydration, protein deficiency), organic salivary gland damage (due to head/ neck radiotherapy, Sjögren's syndrome, HIV infection, aging, salivary glands fibrosis), interaction with neurotransmissions (drugs, central nervous system) [1,2,3].

Xerostomia is defined as the subjective sensation of dry mouth [4]. As a subjective symptom, it can only be diagnosed through a direct questioning of the patient [5].

Along with the changes in the salivary composition and flow, gingivitis and halitosis are relatively common complications in patients undergoing chronic hemodialysis [6, 7], associated with the underlying disease or the results of treatment. In a 2012 study Yu et al. found out that $66.4 \%$ of patients undergoing renal replacement therapy in Taiwan experienced a dry mouth sensation [8]. Although xerostomia is a subjective sensation, its manifestation in patients on hemodialysis can affect their oral health, which in turn often leads to difficulty in chewing, swallowing and speaking. It can also give rise to lesions on the oral mucosa, particularly on the periodontium and the tongue, thus facilitating bacterial and fungal infections, affecting the incidence of dental caries and bad breath [7]. This symptom cluster can seriously influence the social life and further deteriorate the quality of life of patients with end-stage chronic kidney disease (CKD) on chronic dialysis treatment. Citing a 2012 research over 50 million people suffer from CKD with the prospects for ever increasing prevalence across virtually all countries $[9,10,11]$. The alarming incidence rate of CKD justifies the need for a comprehensive study of this group of patients.

The objective of the present study was to determine the prevalence of the subjective sensation of dry mouth in patients with end-stage chronic kidney disease and in healthy control patients, using a questionnaire.

\section{Material AND Methods}

The statistical evaluation of results was carried out using SPSS software package for epidemiological and clinical data analysis (V. 20.00, 2011 Edition). The following statistical methods were applied: descriptive statistics for qualitative data (frequency and percentage tables) and output graphs; cross tabulation for qualitative data; Phi and Cramer's V nominal associations for correlation between qualitative variables; Pearson's chi-square test of independence between qualitative variables and output graphs.

The units of observation were 104 patients, divided into two groups: a Control Group and a Clinical Group.

The Control Group was represented by 34 patients, of whom 20 were female patients (59\%) and 14 males (41\%), with a mean age of 21.76 years (SD $=1.79$ ) The youngest participant was 19 years old, while the oldest patient was 27 years old. The Control Group included patients without any common illness in the functional and physiological equilibrium of the body.

The Clinical Group included 70 patients with end-stage CKD, where 32 were the male patients (46\%) and 38 were the female patients (54\%), undergoing chronic dialysis treatment, with an mean age of 58.9 (SD = 14.46). The youngest participant was 32 years old and the oldest was 89 years of age. 
The clinical study was approved by the Research Ethics Committee at the Medical University - Varna with Protocol No. 55/ 16 June 2016. For the purposes of analysis the subjects in the Clinical Group were divided into 2 age subgroups (relative to the mean age): $32-60$ years old and $61-89$ years old. The first subgroup included 31 individuals (44\%) and the second subgroup - 39 persons (56\%).

The participants were evenly distributed according to gender in age groups. The gender distribution across the age subgroups appeared as follows: 15 men and 16 women were in the first age group, whereas 17 men and 22 women belonged to the second age group. (Table 1)

Table1. Frequency distribution of patients according to their gender and age

\begin{tabular}{|c|c|c|c|c|c|c|}
\hline \multirow{3}{*}{ Gender } & \multicolumn{4}{|c|}{ Age } & \multirow{2}{*}{\multicolumn{2}{|c|}{ Total }} \\
\hline & \multicolumn{2}{|c|}{$32-60$ years old } & \multicolumn{2}{|c|}{61 - 89 years old } & & \\
\hline & Number & $\%$ & Number & $\%$ & Number & $\%$ \\
\hline Male & 15 & 21,4 & 17 & 24,3 & 32 & 45,7 \\
\hline Female & 16 & 22,9 & 22 & 31,4 & 38 & 54,3 \\
\hline Total & 31 & 44,3 & 39 & 55,7 & 70 & 100,0 \\
\hline
\end{tabular}

All participants signed an Informed Consent after being informed in detail of the purpose and terms of participation in the study. The data was collected through a questionnaire, consisting of a basic section (name of participant, gender, and age) and the main question:

\section{Does your mouth feel dry?}

For the purpose of obtaining general information on other subjective oral sensations, the following additional questions were included:

2. Can you feel any taste alteration?

3. Can you feel burning in your mouth?

4. Can you feel a metallic taste in your mouth?

5. Can you feel any loss of taste?

6. Is your mouth sore while eating?

7. Is your mouth sore when swallowing?

\section{Do you have bad breath?}

The answering options needed to be either Yes or No. For the reliability of the data, the researchers relied on the patients' good faith.

\section{RESULTS}

The intraoral examination of all patients from the Clinical Group revealed a pale, unmoist mucosa of the tongue and of the oral cavity as well as the presence of dental plaque and food debris on the soft tissues. Dry nasal mucosa, dry eyes and dry skin were identified, which pointed to the objective evidence of dry mouth as well.

The results obtained indicated that 47 patients (67\%) from the Clinical Group complained of dry mouth, whereas 23 patients (33\%) had no complaints. In the Control Group, 2 healthy volunteers (1.92\%) shared similar complaints, while 32 patients (30.77\%) claimed they did not feel any mouth dryness. The data from the statistical test revealed a statistically significant correlation between xerostomia complaints and the patients' group affiliation $\left(\chi^{2}=34.47, p=0.000\right)$. (Figure 1 ) 
Xerostomia as a Contributing Factor for Taste Distortion in Patients Undergoing Chronic Dialysis Treatment

Fig1. Frequency distribution of patients from the Clinical Group and the Control Group according to their subjective sensation of dry mouth

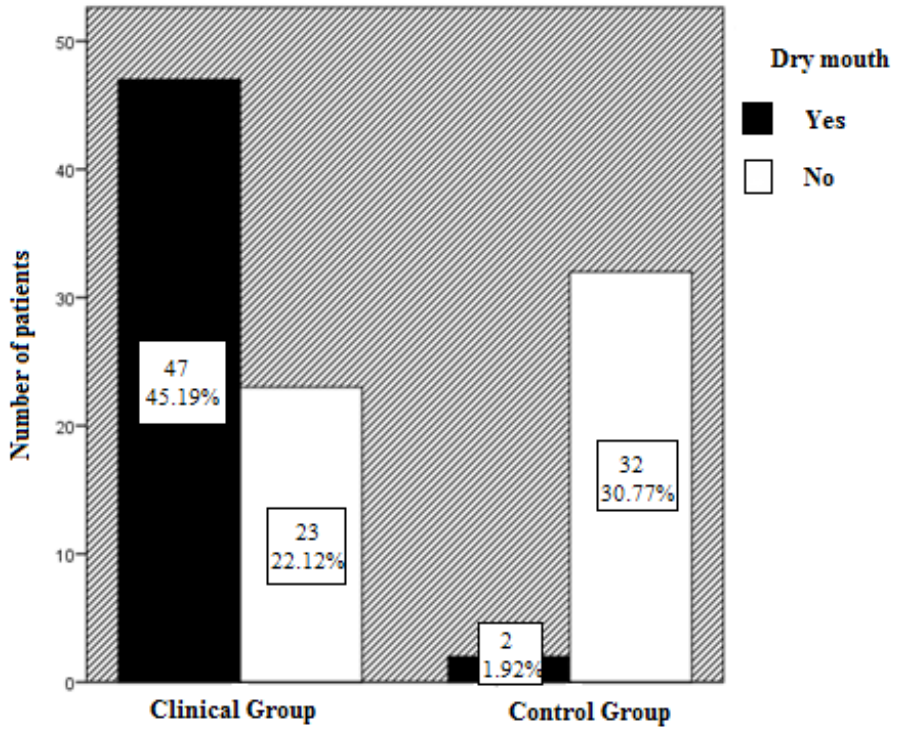

Due to the negligibly small relative proportion of patients in the Control Group $(<2 \%)$, reporting dry mouth, the following results were obtained from the Clinical Group. The data from Pearson's Chi-Square Test showed that gender was not linked to dry mouth complaints $\left(\chi^{2}=0.58, p=0.448\right)$.

A statistically significant dependence between the patients' age and the sensation of dry mouth was not detected, however a tendency towards a greater number of complaints among patients over 60 years of age was apparent $\left(\chi^{2}=2.08, \mathrm{p}=0.149\right)$. (Figure 2)

Fig2. Frequency and percentage distribution of participants from the Clinical Group according to their age and subjective complaints of dry mouth

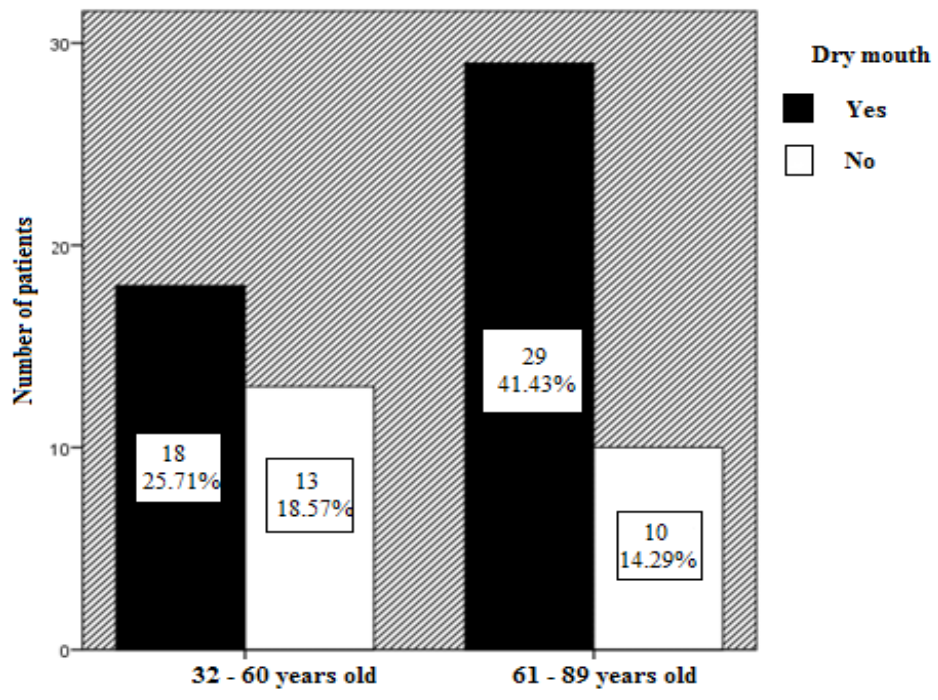

The statistical analysis did not confirm any statistically significant relationship between the duration of hemodialysis and dry mouth complaints $\left(\chi^{2}=0.87, \mathrm{p}=0.352\right)$. (Figure 3 ) 
Fig3. Frequency and percentage distribution of participants from the Clinical Group according to the duration of hemodialysis and their subjective complaints of dry mouth

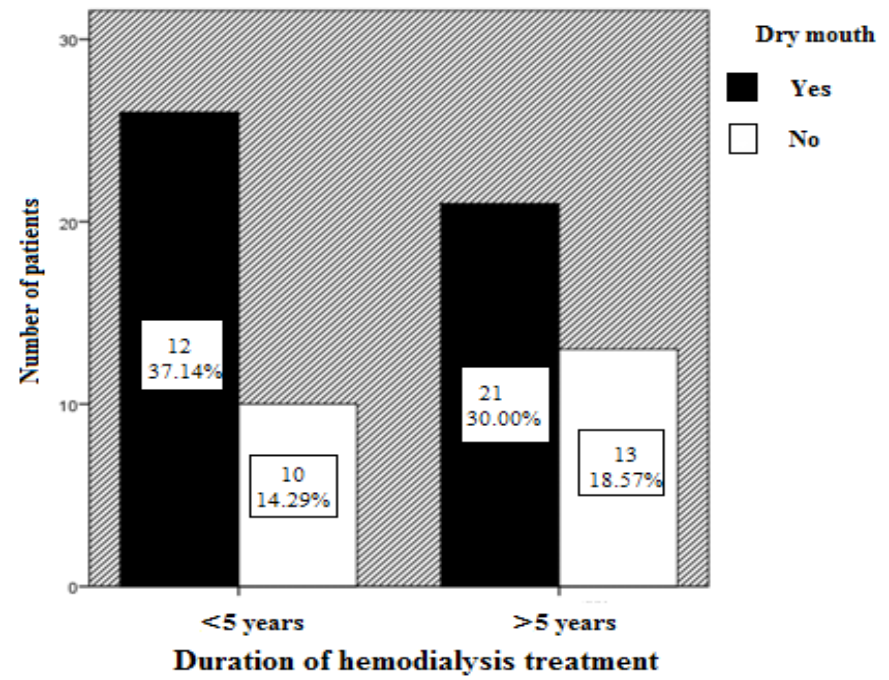

For the purposes of precise analyses, the Chi-Square test of independence was applied individually for both age subgroups. The data derived from the patients aged 32-60 years indicated that there was no statistically significant correlation between the duration of hemodialysis and the subjective complaints of dry mouth $\left(\chi^{2}=\right.$ $0.68, p=0.409$ ), however a tendency towards a greater number of dry mouth complaints among patients over 60 years of age was evident. Conversely, the correlation between the duration of hemodialysis and dry mouth in patients aged $61-89$ years proved to be statistically significant $\left(\chi^{2}=3.81, p=0.05\right)$. The analyses revealed that fewer patients in the Clinical group, who had underwent over 5 years of treatment, reported the symptom of dry mouth.

Table2. Correlations between dry mouth complaints and other subjective complaints of patients from the Clinical Group

\begin{tabular}{|c|c|c|c|c|c|c|c|c|}
\hline & 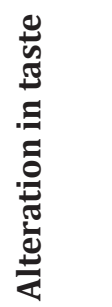 & 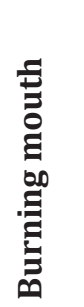 & 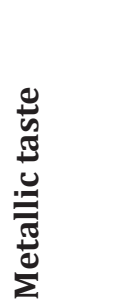 & 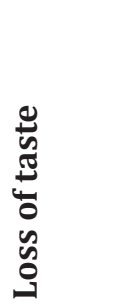 & 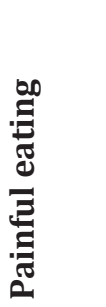 & 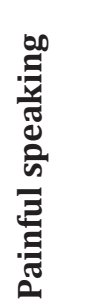 & 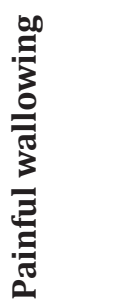 & 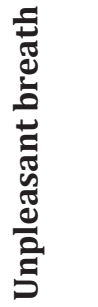 \\
\hline $\begin{array}{l}\text { Dry mouth in all } \\
\text { patients }\end{array}$ & $0.28^{*}$ & 0.20 & $0.36^{* *}$ & $0.32^{* *}$ & $0.26^{*}$ & 0.06 & $0.30 *$ & $0.23^{*}$ \\
\hline $\begin{array}{l}\text { Dry mouth in patients } \\
\text { with < } 5 \text { years of } \\
\text { hemodialysis }\end{array}$ & $0.37^{*}$ & 0.25 & $0.37^{*}$ & 0,28 & $0.33^{*}$ & 0.11 & 0.22 & $0.40^{*}$ \\
\hline $\begin{array}{l}\text { Dry mouth in patients } \\
\text { with > } 5 \text { years of } \\
\text { hemodialysis }\end{array}$ & 0.21 & 0.10 & 0.33* & $0.38^{*}$ & 0.16 & -0.06 & $0.36^{*}$ & 0.04 \\
\hline
\end{tabular}

Key: * Significance threshold at 0.05

** Significance threshold at 0.01 as per Cohen [12] 
Using Phi coefficient for nominal measures of association between two binary variables, statistical dependences were established between dry mouth complaints and other subjective complaints related to taste. The data pointed to significant positive correlations between dry mouth and the following symptoms: alteration in taste, metallic taste, loss of taste, soreness while eating and swallowing as well as unpleasant breath. Further analysis of the correlation between dry mouth and other subjective complaints indicated deviations depending on the duration of the renal replacement therapy. (Table 2)

The analysis of data showed that there was a statistically significant correlation between the sensation of dry mouth and taste alteration as well as between dry mouth and soreness while eating in patients on hemodialysis treatment $<5$ years. There was a statistical dependence between xerostomia and taste loss as well as between xerostomia and painful swallowing in patients who had been on renal therapy $>5$ years. The observations showed the presence a statistical dependence between dry mouth and bad breath in patients who had been treated $<5$ years.

\section{DISCUSSION}

Xerostomia appears to be a common complaint among CKD patients, while it may not be experienced equally across individuals. This is evident in the lack of the subjective feeling of oral dryness in 23 patients, despite the terminal stage of the disease and the hemodialysis treatment. The symptoms typically shared involved difficulty in speaking, mastication and swallowing. The study data showed statistically significant dependencies between the feeling of dry mouth and the symptoms listed above. Patients reported the need to moisten the mouth, suckling on a candy, taking liquids to assist swallowing dry food. It is necessary to assess the extent of discomfort incurred while chewing, swallowing and speaking and to prove the presence of taste alteration, burning mouth syndrome, etc. $[13,14]$. Furthermore, it is imperative to evaluate the dietary restrictions that xerostomia may lead to in view of the potential macro- and micro-nutrient deficiency. To date dental research literature supplies only insufficient unilateral data on the subject. For instance, the imposed common restraint from bakery products (bread, toast, crackers) has been underinvestigated. Some authors failed to prove a significant difference in either nutritional preferences [15] or the intake of fiber, carbohydrates, vitamins, which reflects the natural preference to fruits and the avoidance of pastries [16]. Other researchers emphasize on the considerably lower intake of whole grain products and the increased intake of fruit, including canned fruit [17]. Some leading international organizations such as FDA, the National Institute of Dental and Craniofacial Research, Mayo Clinic and the US National Library of Medicine and MedlinePlus recommend avoiding brittle and hard bread, strong spices and acidic flavors like vinegar, lemon, extremely salty or sweet products, dried meat, which are particularly challenging for chewing. Observing such restrictions without the advice of a nutritionist would inevitably affect the nutritional value of the daily menu. The duration of the renal replacement therapy of CKD patients can impact the manifestation of a number of oral symptoms, including xerostomia [18, 19, 20, 21, 22]. They typically result from the metabolic and physiological changes inherent to the disease. Scientific literature suggests evidence of the relationship between the duration of the dialysis treatment and the development of oral lesions $[18,23]$. The present study of patients suffering from renal impairment outlined a tendency toward increasing complaints of dry mouth in the cases of hemodialysis treatment lasting $>5$ years $\left(\chi^{2}=3.81, p=0.05\right)$. The data implied that certain patients tend to get accustomed to the dry mouth symptom over the course of time. This is evident in the lack of the subjective feeling of oral dryness in many patients aged $61-89$ years. Xerostomia is a socially significant disease due to its increasingly recognized link to multiple caries, periodontal complications and rapid tooth loss. The psychological consequences of xerostomia are real and can occasionally create social anxiety and shame with refusal to leave home, talk for a long time and eat out. 


\section{CONCLUSION}

Chronic kidney disease has a lasting and significant impact on patients' quality of life and poses a serious health and social problem. Although xerostomia is a subjective sensation, its manifestation in patients on hemodialysis can affect their oral health. The issue is still relevant with the increasing incidence of CKD and its relation the quality of life of this group of patients.

\section{REFERENCES}

1. Bergdahl M, Bergdahl J. Low unstimulated salivary flow and subjective oral dryness: Association with medication, anxiety, depression, and stress. J Dent Res 79:1652-1658, 2000.

2. Daniels TE, Fox PC. Salivary and oral components of Sjogren's syndrome. Rheumatic diseases clinics of North America, 1992, 18.3: 571-589.

3. Pink R, Simek J, Vondrakova J, Faber E, Michl P. et al. Saliva as a diagnostic medium. Biomed Pap Med Fac Univ Palacky Olomouc Czech Repub, 2009, 153.2: 103-110.

4. Fox PC, Busch KA, Baum BJ. Subjective reports of xerostomia and objective measures of salivary gland performance. JAmDent Assoc115:581-584, 1987.

5. Hopcraft MS, Tan C. Xerostomia: an update for clinicians. Australian dental journal, 2010, 55.3: 238-244.

6. Curtin RB, Bultman DC, Thomas-Hawkin C, Walters BAJ, Schatell D. Hemodialysis patients' symptom experiences: effects on physical and mental functioning. Nephrol. Nurs. J. 2002, 29 (6), 562.

7. Bossola M, Tazza L. Xerostomia in patients on chronic hemodialysis. Nat. Rev. Nephrol. 2012, 8 (3), 176182. doi:http://dx.doi.org/10.1038/nrneph.2011.218.

8. Yu IC, Huang JY, Tsai YF. Symptom cluster among hemodialysis patients in Taiwan. Appl. Nurs. Res. 2012, 25 (3), 190-196. doi:http://dx.doi.org/10.1016/j. apnr.2010.11.002.

9. Corrao AM, Lisi G, Di Pasqua G. et al. Serum cystatin C as a reliable marker of changes in glomerular filtration rate in children with urinary tract malformations. J Urol, 175, 2006, 303-309.

10. Levin A, Hemmelgarn B, Culleton B. et al. Guidelines for the management of chronic kidney disease, Serum cystatin C and prediabetes in non-obese US adults. CMAJ, 179, 2008, 1154-1562.

11. Stevens LA, Levey AS. Chronic kidney disease in the elderly - how to assess risk. NEJM, 352, 2005, 21222124.

12. Cohen J. Statistical Power Analysis for the Behavioral Sciences, 2nd ed. 1988, Hillsdale, NJ: Erlbaum.

13. Palacios-Sanchez MF, Jordana-Comin X, Garcia-Sivoli CE. Burning mouth syndrome: a retrospective study of 140 cases in a sample of Catalan population, Med Oral Patol Oral Cir Bucal, 10 (5) (2005), pp. 388-393.

14. Soares MS, Chimenos-Kustner E, Subira-Pifarre C, Rodriguez de Rivera-Campillo ME, Lopez-Lopez J. Association of burning mouth syndrome with xerostomia and medicines. Med Oral Patol Oral Cir Bucal, 10 (4) (2005), pp. 301-308.

15. Sheiham A, Steele JG, Marcenes W. et al. The impact of oral health on stated ability to eat certain foods; findings from the National Diet and Nutrition Survey of Older People in Great Britain. Gerodontology. 1999;16(1):11-20.

16. Cermak JM, Papas AS, Sullivan RM, et al. Nutrient intake in women with primary and secondary Sjogren's syndrome. Eur J Clin Nutr. 2003;57:328-334. 
Xerostomia as a Contributing Factor for Taste Distortion in Patients Undergoing Chronic Dialysis Treatment

17. Quandt SA, Savoca MR, Leng X, Chen H, Bell RA. Et al. Dry mouth and dietary quality in older adults in North Carolina. Journal of the American Geriatrics Society. 2011 Mar 1;59(3):439-45.

18. Block GA, Martin KJ, De Francisco AL. Cinacalcet for secondary hyperparathyroidism in patients receiving hemodialysis. N Engl J Med. 2004; 350:1516-1525.

19. Chi AC, Neville BW, Krayer JW, Gonsalves WC. Oral manifestations of systemic disease. Am FamPhysician 2010;82:1381-1388.

20. Souza CM, Braosi A, Luczyszyn S. et al. Oral health in Brazilian patients with chronic renal disease. Rev Méd Chile 2008; 136: 741-746.

21. Nenova- Nogalcheva A, Konstantinova D. Halitosis in patients with end-stage chronic kidney disease undergoing chronic dialysis treatment. IJSR 2016; Volume 5 Issue 12, 875-878.

22. Sobrado MJS, Tomás CI, Loureiro A et al. Oral health status in patients with moderate-severe and terminal renal failure. Med Oral Patol Oral Cir Bucal. 2007 ;12(4):E305-10.

23. De Rossi SS, Glick M. Dental considerations for the patient with renal disease receiving hemodialysis. J Am Dent Assoc. 1996; 127(2):211-219.

Citation: D. Konstantinova, A. Nenova-Nogalcheva, P. Pechalova, R. Pancheva, D. Andonova, "Xerostomia as a Contributing Factor for Taste Distortion in Patients Undergoing Chronic Dialysis Treatment". American Research Journal of Dentistry; 1(1): 1-8.

Copyright (C) D. Konstantinova, A. Nenova-Nogalcheva, P. Pechalova, R. Pancheva, D. Andonova, This is an open access article distributed under the Creative Commons Attribution License, which permits unrestricted use, distribution, and reproduction in any medium, provided the original work is properly cited. 